\title{
Análise Parasitológica de Caldos de Cana Comercializados em Feiras Livres em uma Cidade no Interior da Bahia
}

\author{
Frederico Azevedo Neves Pina ${ }^{1}$; Marcelo José Costa Lima Espinheira ${ }^{2}$; Flávio Mendes de Souza ${ }^{3}$
}

\begin{abstract}
Resumo: As parasitoses no Brasil ainda constituem um grande problema de saúde pública sendo mais frequentes em populações com níveis socioeconômicos mais baixos. O caldo de cana é uma bebida de alto valor energético, e amplamente consumidas pela população, principalmente nas regiões de clima tropical e subtropical e durante o verão. A ausência de informações dos manipuladores e as condições higiênico-sanitárias precárias dos locais de venda permitem que este alimento seja foco de diversas contaminações microbiológicas e parasitológicas. Portanto, esse estudo objetiva verificar a contaminação por parasitas nos caldos de cana das feiras livres de Vitória da Conquista-Ba, pela analise parasitológica do caldo de cana, através do método de sedimentação espontânea Hoffman, Pons e Janer (1934). Dessa forma, foram analisadas 18 amostras de caldo de cana, de modo que em 72,2\% delas havia cistos de Entamoeba coli, em 38,8\% foram vistos ovos de Ascaris lumbricoides, em 27,7\% encontrou-se cistos de Giardia lamblia, e em 5,5\% foram identificados oocistos de Isospora belli. Neste cenário, com a pesquisa, foi possível notar que os cuidados higiênico-sanitários por parte dos manipuladores de alimentos foram baixos.
\end{abstract}

Palavras - chave: Contaminação; Parasitas Intestinais; Alimentos.

\section{Parasitological Analysis of Sugar Cane Juice Marketed in free fairs in a City of the Interior of Bahia}

\begin{abstract}
The parasitic diseases in Brazil are an important public health's problem, being more common in the populations who have the lowest socioeconomic levels. The cane broth is a beverage with high energetic content, end being widely consumed by the population, mainly at the tropical and subtropical regions and during the summer. The absence of information by the manipulators and the precarious hygienic-sanitary conditions at the places of sale allow that this food be a focus of various microbiological and parasitological contamination. Therefore, this study objectify to check the contamination by parasites in the cane broth from the fairs in Vitoria da Conquista-Ba, by using a parasitological analysis of the cane broth, with the Hoffman, Pons and Janer spontaneous sedimentation method (1934). So 18 cane broth's samples were analyzed, and were found cysts of Entamoeba coli in $72,2 \%$ of this, eggs of Ascaris lumbricoides in 38,8\%, cysts of Giardia lamblia in $27,7 \%$ and oocysts of Isospora belli in 5,5\%. Consequently, with the research, was possible to note that the hygienic-sanitary cares by the manipulators of foods were low.
\end{abstract}

Key words: Contamination, Intestinal Parasites; Foods

\footnotetext{
${ }^{1}$ Frederico Azevêdo Neves Pina, graduando em Farmácia pela Faculdade Independente do Nordeste.

Contato: nevessazevedo@hotmail.com;

${ }^{2}$ Marcelo José Costa Lima Espinheira, Biólogo graduado pela Universidade Católica de Salvador - UCSAL, Pós graduado em Metodologia e Gestão do Ensino Superior pela Faculdade Independente do nordetse - FAINOR, Meste em Religião/Educação na linha de pesquisa sobre Ética e gestão pela faculdade EST. Docente da FAINOR, FTC e UNINASSAU.

${ }^{3}$ Flávio Mendes de Souza, graduado em química pela Universidade estadual de Santa Cruz - UESC, mestre em química pela Universidade Estadual da Bahia - UESB. Docente da Faculdade Independente do Nordeste - FAINOR.
} 


\section{Introdução}

A produção da cana de açúcar (Saccharum spp.) é considerada uma das plantações mais significativas das regiões tropicais e subtropicais. Ela é uma gramínea originária do Sudeste Asiático, composta pelo bagaço, que é rico em fibras, e pelo caldo, que é um líquido composto por água, açúcares, cinzas e materiais nitrogenados, obtido por meio da moagem e deve ser consumido imediatamente. Os carboidratos do caldo são bastante digestíveis, e por ser rico em carboidratos e pobre em proteínas, ele é considerado como uma bebida extremamente energética (PRADO, 2010).

O comércio de alimentos nas ruas, nas regiões subdesenvolvidas, teve um elevado crescimento nos últimos 10 anos, pois se transformou em uma opção de emprego, mas as deficientes condições higiênico-sanitárias dos locais onde são comercializados permitem que, associados à ausência de treinamento e conhecimento dos vendedores, esses alimentos ocasionem riscos à saúde da população, devido à facilidade de contaminação dos alimentos por microrganismos (PRADO, 2010). Os estabelecimentos de preparo e comércio dos alimentos exercem um papel importante no que determina a qualidade da alimentação da população, sobretudo urbana, que em virtude do tempo acessível para a preparação e a ingestão dos alimentos, preferem refeições mais rápidas, tanto na obtenção e preparo quanto no consumo (CRISTINE, 2006).

A prevalência de doenças transmitidas por alimentos está associada aos hábitos de higiene precários dos manipuladores com a falta do controle ambiental e por não haver um local adequado para a venda dos mesmos (ROSA, 2010). A produção do caldo de cana baseia-se em um pequeno número de procedimentos, no entanto, é um processo crítico, que pode favorecer a inserção de microrganismos ou a multiplicação daqueles já presentes no vegetal. Os resíduos do processo são frequentemente deixados próximo a moenda, atraindo animais e insetos, razões que podem auxiliar na ocorrência de Enfermidades Transmitidas por Alimentos (ETA) (PRADO, 2010). Os manipuladores de alimentos também são responsáveis pela disseminação onde a maioria dos quiosques do caldo de cana estão localizadas em regiões que não há um sistema de abastecimento de água tratada, comprometendo a higienização correta das mãos e dos equipamentos para preparo do caldo, além do mais, diversos procedimentos inadequados do ponto de vista higiênico são observados, como a manipulação de dinheiro, uso de panos

sujos, dentre outros (CARVALHO, 2011). Essas doenças são uma grande dificuldade para a

860 Id on Line Rev. Mult. Psic. V.12, N. 40. 2018 - ISSN 1981-1179 Edição eletrônica em http://idonline.emnuvens.com.br/id 
saúde pública, pois estima-se que milhões de pessoas estejam acometidas por elas, tanto em países desenvolvidos quanto em desenvolvimento, pois afeta tanto em nível da saúde das populações quanto ao desenvolvimento da economia destes países, porque geram incapacidade laboral e elevam os custos em tratamentos e hospitalizações (PRADO, 2010).

Visando diminuir os problemas de contaminação e possíveis veiculações de DTAs, a Agência Nacional de Vigilância Sanitária (ANVISA) criou a Resolução RDC nº 218, de 29 de julho de 2005. Nesta está disposto o Regulamento Técnico de Procedimentos HigiênicoSanitários para Manipulação de Alimentos e Bebidas Preparadas com Vegetais, estando também incluído o caldo de cana (BRASIL, 2005).

Por conta da falta de fiscalização, na maioria das vezes os comerciantes não obedecem às leis (SANTOS et al., 2010). Também há falta de informações e treinamento acerca dos cuidados higiênicos sanitários que devem ser realizadas durante todo o processo, desde a manipulação da matéria prima até o produto final. Se estes procedimentos forem corretamente implantados na cadeia produtiva diminuem em mais de 50\% os riscos do contágio com DTAs (PEREIRA et al., 2009).

Portanto, como observa-se um alto consumo de caldo de cana na cidade, principalmente no verão, este estudo possui como objetivo verificar a incidência de parasitas presentes no caldo de cana e os possíveis fatores associados a esta contaminação.

\section{Metodologia}

O estudo foi realizado nas feiras livres em Vitória da Conquista - Bahia, onde variados perfis de consumidores transitam diariamente. Nesses locais, há comercialização de frutas, carnes, verduras entre outros tipos de itens alimentícios, incluindo o caldo de cana.

O município de Vitória da Conquista está localizado no sudoeste da Bahia e é classificado como o terceiro maior do estado, distando em aproximadamente $509 \mathrm{Km}$ de Salvador. Segundo dados do IBGE - Instituto Brasileiro de Geografia e Estatística, Vitória da Conquista possui população de aproximadamente 348.718 habitantes (IBGE, 2017).

As amostras de caldo de cana foram coletadas em seis (06) pontos de vendas, comercializadas em três (03) feiras livres escolhidas de forma aleatória em Vitória da Conquista - Bahia, entre os dias 10 de março a 24 de março de 2018. 
Foram coletadas três (03) amostras de $200 \mathrm{ml}$ por estabelecimento $(\mathrm{n}=18)$, em frascos estéreis de $250 \mathrm{ml}$, previamente identificados (A1 - A6), de acordo com a sequência de coleta. $\mathrm{O}$ acondicionamento foi feito em caixas isotérmicas e os materiais foram encaminhados imediatamente ao laboratório de parasitologia da Faculdade Independente do Nordeste (FAINOR). As amostras foram processadas pelo método de sedimentação espontânea Hoffman, Pons e Janer (1934), que além de ser um método de baixo custo e fácil execução tem como objetivo a identificação de ovos e larvas de helmintos, cistos e oocistos de protozoários (REY, 2001).

O material coletado foi homogeneizado e transferido para um cálice de Hoffman de 250 ml, parte do sedimento foi colocada em uma lâmina corada com lugol, para uma melhor visualização, e uma lamínula foi sobreposta. Assim, a análise foi feita em triplicata, utilizando um microscópio óptico em objetivas de 10X e 40X.

\section{Resultados e Discussão}

Após as análises parasitológicas, os resultados obtidos mostraram que 77,8\% (n=14) das amostras foram positivas no período coletado, sendo identificadas 72,2\% ( $\mathrm{n}=13)$ de cistos de Entamoeba coli, 38,8\% ( $\mathrm{n}=7)$ de ovos de Ascaris lumbricoides, 27,7\% ( $\mathrm{n}=5)$ de cistos de Giardia lamblia e 5,5\% (n=1) de oocistos de Isospora belli, demostrando que há uma grande deficiência higiênico-sanitária nos estabelecimentos que foram coletados as amostras.

Tabela 1. Enteroparasitas encontrados nas feiras livres em 10 de março de 2018.

\begin{tabular}{l|ccccccc} 
ENTEROPARASITAS: & A1 & A2 & A3 & A4 & A5 & A6 \\
\hline Cistos de Entamoeba coli & X & - & X & - & X & - \\
Ovos de Ascaris lumbricoides & - & - & - & - & X & - \\
Cistos de Giardia lamblia & X & - & X & - & - & - \\
Oocistos de Isospora beli & - & - & - & - & X & - \\
\hline
\end{tabular}


Tabela 2. Enteroparasitas encontrados nas feiras livres em 17 de março de 2018.

$\begin{array}{lllllll}\text { ENTEROPARASITAS: } & \text { A1 } & \text { A2 } & \text { A3 } & \text { A4 } & \text { A5 }\end{array}$

\begin{tabular}{l|cccccc|}
\hline Cistos de Entamoeba coli & $\mathrm{X}$ & - & $\mathrm{X}$ & - & $\mathrm{X}$ & $\mathrm{X}$ \\
Ovos de Ascaris lumbricoides & $\mathrm{X}$ & - & - & - & $\mathrm{X}$ & - \\
Cistos de Giardia Lamblia & $\mathrm{X}$ & $\mathrm{X}$ & - & - & - & - \\
Oocistos de Isospora beli & - & - & - & - & - & - \\
\end{tabular}

Tabela 3. Enteroparasitas encontrados nas feiras livres em 24 de março de 2018.

\begin{tabular}{l|ccccccr} 
ENTEROPARASITAS & A1 & A2 & A3 & A4 & A5 & A6 \\
\hline Cistos de Entamoeba coli & X & X & X & X & X & X \\
Ovos de Ascaris lumbricoides & X & X & - & X & X & - \\
Cistos de Giardia lamblia & - & X & - & - & - & - \\
Oocistos de Isospora beli & - & - & - & - & - & - \\
\end{tabular}

As amostras tiveram uma maior prevalência de cistos de Entamoeba coli, cuja forma de transmissão é de forma direta (indivíduo para indivíduo) ou pela ingestão de água e alimentos contaminados (COSTA, 2012). De acordo os estudos de Jung et al. (2014), foi encontrada também presença de E.coli em alfaces, demonstrando que existe o contato com fezes de humanos ou de animais nos locais de cultivo, e ainda que este parasita não seja patogênico no homem, isso se torna um indicativo da falta de higienização de quem manipula essas plantas, demonstrando a qualidade precária da higiene dos alimentos nos locais de cultivo.

Durante o período de coleta, além dos cistos de Entamoeba coli foram encontrados também ovos de Ascaris lumbricoides nas amostras A5 em todos os dias da coleta. Em um dos estudos de Oliveira et al. (2009), o percentual desse helminto em uma análise realizada na população de Goioerê - PR foi de 39,2\%, e esta elevada taxa de ocorrência pode ser justificada 
pelo fato de serem bem resistentes às condições do meio ambiente, sendo facilmente dissipados através de poeira, chuvas, ventos, insetos e veiculados principalmente pela água. A sua incidência pode ser causada pelo alto número de pessoas infectadas, já que, de acordo Centers for Disease Control and Prevention (2018), há uma estimativa que 807 milhões a 1,2 bilhões de pessoas estejam infectadas. Esse helminto pode causar irritação na parede intestinal, alterações respiratórias e também levar à obstrução do intestino, assim como o verme também consome grande quantidade de proteínas, carboidratos e algumas vitaminas do hospedeiro (NEVES; MELO; LINARDI, 2005).

A Giardia lamblia é um protozoário flagelado que possui duas formas evolutivas: cisto e trofozoíto. A contaminação ocorre pela ingestão da forma cística, que ao chegar ao intestino passa para a forma trofozóita, que está presente na mucosa do duodeno, onde ele se multiplica. A sua transmissão está relacionada com as más condições de higiene, com a contaminação de água e alimentos e com o fato de os cistos contaminantes poderem resistir às condições do meio ambiente por meses. Os sintomas que se expressam clinicamente no infectado mantém direta relação com a quantidade de cistos que a pessoa ingeriu, com as condições imunológicas do hospedeiro e com a duração da doença. A manifestação clínica da giardíase mais comum é a diarreia fétida (SILVA, 2017). Pereira (2016) realizou análise em saladas pré-embaladas, e a presença do protozoário foi identificada em $4,16 \%$ das amostras, e mesmo que este não seja um indicativo muito elevado, permite inferir que não houve a higienização durante o preparo das saladas, que assim como o caldo de cana são alimentos consumidos crus.

$\mathrm{Na}$ amostra A5, coletada no dia 10 de março, foram encontrados oocistos de Isospora beli, sendo classificados como coccídeo, pois o protozoário possui mais de uma espécie, e a Isospora beli é a única reconhecida como patogênica. A sua transmissão acontece pela ingestão de água e alimentos contaminados com os oocistos. Por possuir uma membrana resistente, os oocistos podem resistir por meses às condições do meio ambiente. Os sintomas da doença incluem diarreia, cefaleias, febre, dor abdominal, vômito, desidratação e perda de peso, principalmente em pessoas imunocomprometidas (LIMA; SAMPAIO, 2016). 
Gráfico 1. Percentual de parasitas encontrados nos caldos de cana nos dias 13, 17 e 24 de março de 2018

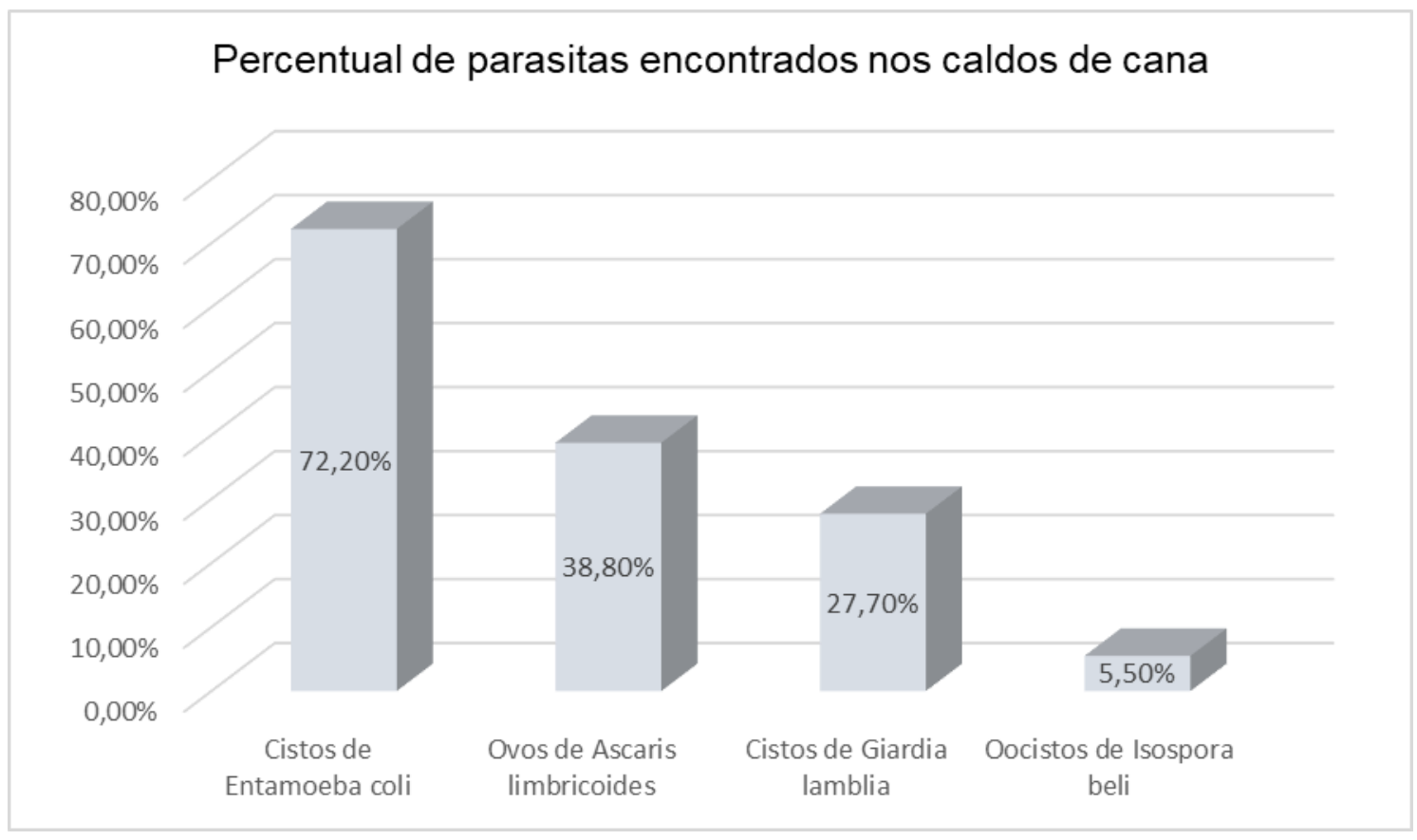

Essa quantidade de parasitas encontrados pode ser justificada por vários fatores, que dentre eles é possível verificar que os estabelecimentos não cumpriam às normas estabelecidas pela RDC 216, de 15 de setembro de 2004, que dispõe sobre o Regulamento Técnico de Boas Práticas para Serviços de Alimentação, nem à RDC 218, de 29 de julho de 2005, que dispõe sobre o Regulamento Técnico de Procedimentos Higiênico-Sanitários para Manipulação de Alimentos e Bebidas preparados com vegetais. Por ser um alimento geralmente consumido cru, a cana-de-açúcar não passa por nenhum tratamento térmico para gerar o caldo e diminuir a incidência de patógenos que não sobrevivem ao calor, por isso a higienização prévia das mãos dos manipuladores sempre que forem preparar a bebida é crucial, já que o indivíduo potencialmente parasitado pode representar uma grande fonte de transmissão. (AZEVEDO et al., 2014).

Os estudos de Lopes et. al. (2007), relataram que entre os comerciantes são adotados diversos procedimentos inadequados do ponto de vista higiênico, como a manipulação de dinheiro, uso de panos sujos para limpeza, além de comercializarem produtos encontrados prontos para a venda sem refrigeração e higienização adequada. 
A RDC 218, de 29 de julho de 2005 dispõe que os manipuladores devem adotar medidas que diminuem os riscos de contaminação, como descrito no item 4.2.1. "Adotar procedimentos que minimizem o risco de contaminação dos alimentos e bebidas preparados, por meio da lavagem das mãos e pelo uso de luvas descartáveis ou utensílios.” (BRASIL, 2005). De acordo a RDC, notou-se que dos estabelecimentos coletados apenas 33,3 \%, utilizavam luvas descartáveis durante o período de coleta, e nestes que utilizavam, não foi observada a troca das mesmas entre um preparo e outro.

Gráfico 2. Utilização de luvas durante o preparo do caldo de cana, durante os dias 10 , 17 e 24 de março de 2018.

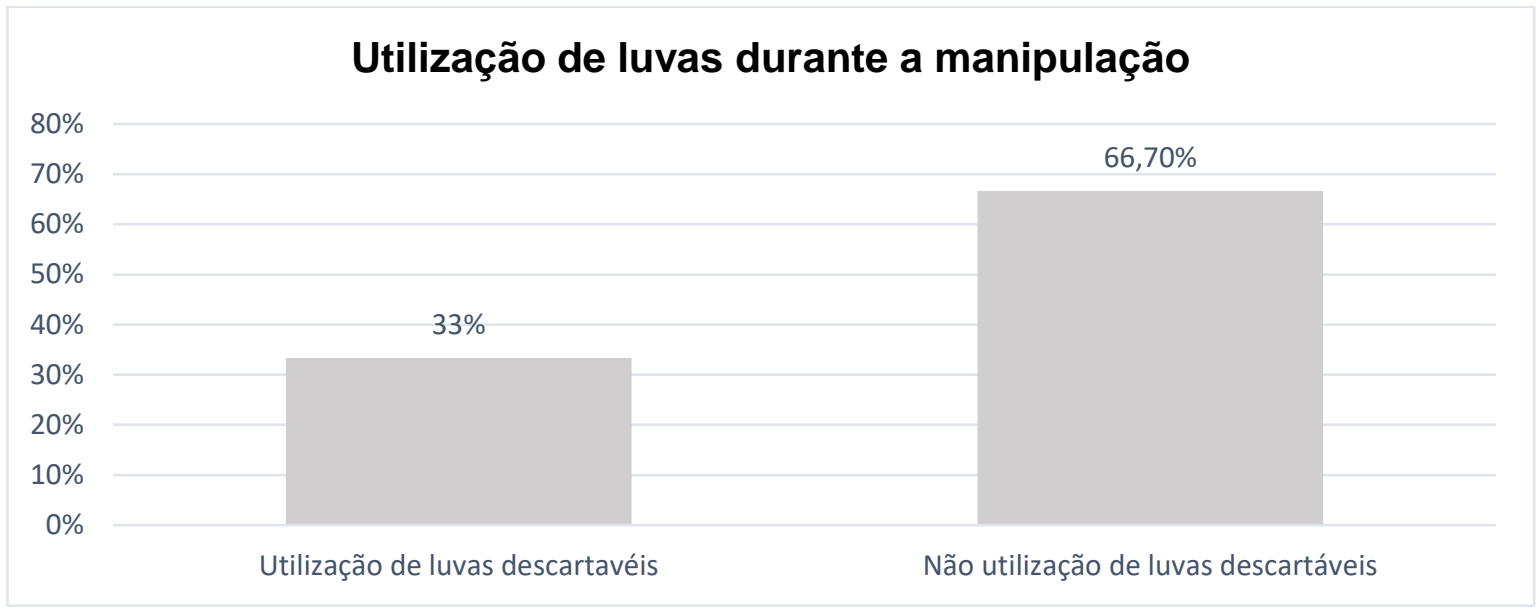

De acordo o parágrafo 4.3. da RDC no 218 de julho de 2005, o local deve ser protegido para evitar o acesso de vetores e pragas (BRASIL, 2005). No estudo feito, foi observado que em $100 \%$ (n=6) dos locais visitados para venda do caldo de cana não havia condições adequadas para a produção seguida da comercialização de produtos alimentícios, pois foram notados fatores que auxiliam a proliferação dos parastisas, como a existência de esgoto circundante, a exposição desprotegida da matéria-prima, a presença de moscas, e a não higienização do local de preparo das bebidas. No estudo feito por Cristine et. al. (2006), realizado em São Paulo, que avaliou as condições do comércio do caldo de cana, verificou resultados semelhantes quanto ao local de preparo de alimentos, no qual o estudo mostrou que $78 \%$ dos estabelecimentos não apresentavam proteção para evitar o acesso de pragas e vetores. 
Além do monitoramento das condições higiênicas do solo, da água de irrigação, transporte e manipulação, é considerável ressaltar a importância do processo de educação sanitária, como rede de esgoto e fossas para todos, concedendo uma melhor condição de higiene para manipuladores e população que consome tais alimentos (ESTEVES; FIGUEIRÔA, 2009) (LUZ et al., 2017).

\section{Considerações Finais}

A realização do presente trabalho permitiu que fosse traçado um perfil da incidência da contaminação parasitária de uma bebida bastante consumida na cidade de Vitória da Conquista$\mathrm{Ba}$, e assim foram encontradas consideráveis quantidades de patógenos em grande parte das amostras. Sendo assim, inferiu-se que a precária condição de salubridade presente no comércio de alimentos continua consagrando como uma das principais vias de transmissão das doenças parasitárias, que permanecem como desafio no âmbito da saúde pública no Brasil.

Diversos aspectos sociais estão entrelaçados na gênese da transmissão dessas doenças à população, a exemplo da escassez de tempo contemporânea individual e coletiva para que a população consiga produzir seu próprio insumo ou da carência na capacitação e no próprio investimento em técnicas que melhorem a higiene no manejo pelos manipuladores do alimento. Sendo assim, a abordagem desses determinantes pode efetivar uma diminuição dessas taxas de contaminação, diminuindo a veiculação do produto nessas condições e evitando uma das vias de propagação das doenças consequentes à ingestão destes patógenos.

Portando, considerando o reconhecimento da importância da comercialização de alimentos na rua associado à necessidade de garantir que o consumo por parte da população seja seguro, algumas medidas deveriam ser adotadas para amenizar o problema.

Assim, resoluções como a aplicação de normas sanitárias adequadas a esta modalidade de comércio, realizadas em congruência com uma rígida e efetiva fiscalização, além da oferta de cursos para os vendedores, orientado pela Vigilância Sanitária, somada a tomadas de medidas governamentais de regularização e controle da atividade poderiam auxiliar na amenização da incidência das doenças parasitárias e das suas importantes repercussões no cenário brasileiro de saúde pública. 


\section{Referências}

AZEVEDO, A. M. F. DE et al. Análise parasitológica do caldo de cana e das condições higiênico-sanitárias do seu comércio no centro da cidade de Fortaleza, Ceará. Nutrivisa: Revista de Nutrição e Vigilância em Saúde, v. 1, n. 2, p. 20-25, 2014.

BRASIL. Instituto Brasileiro de Geografia e Estatistica. Censo demográfico, 2017. Disponível em: <www.ibge.gov.br.> Acesso em: 28 out. 2017.

BRASIL. Resolução RDC no 12, de 02 de Janeiro de 2001. Regulamento Técnico sobre padrões microbiológicos para alimentos. Ministério da Saúde, v. 53, n. 9, p. 1689-1699, 2001. Disponível em: <http://www.anvisa.gov.br>. Acesso em: 04 abril 2018.

BRASIL. Resolução RDC $n^{\circ}$ 2016, de 15 de setembro de 2014. Dispões sobre Regulamento Técnico de Boas Práticas para Serviço de Alimentação. Ministério da Saúde, p. 1-14, 2004. Disponível em: <http://www.anvisa.gov.br>. Acesso em: 04 abril 2018.

BRASIL. Resolução RDC $N^{\circ} 218$, de 29 de julho de 2005. Dispõe sobre o Regulamento Técnico de Procedimentos Higiênico-Sanitários para Manipulação de Alimentos e Be-bidas Preparados com Vegetais. Ministério da Saúde, p. 8, 2005. Disponível em: 〈http://www.anvisa.gov.br>. Acesso em: 04 abril 2018.

CARDOSO, A.V.; LESCANO, S.A.; AMATO NETO, V.; GAKIYA, É.; SANTOS, S.V. Survival of Trypanosoma cruzi in sugar cane used to prepare juice. Revista do Instituto de Medicina Tropical de São Paulo, v.48, n.5, p.287-289, 2006.

CARVALHO, F. A. L.; MOLINARI, R. F.; CONDÉ, M. J. G.; LOPES, C. L.; OLIVEIRA, M. F. F.; RODRIGUES, F. C. Avaliação premilinar da qualidade microbiológica do caldo de cana comercializado por ambulantes na cidade de Viçosa, MG, Brasil. ANAIS III SIMPAC, v. 3, p. 146-151, 2011.

CENTERS FOR DISEASE CONTROL AND PREVENTION (Atlanta). Parasites: Ascariasis. 2013. Disponível em: <http:// www.cdc.gov/parasites/ascariasis/>. Acesso em: 4 abril 2018.

COSTA, J. R. B. Ocorrência de enteroparasitos em amostras de alface ( lactuca sativa l. ) comercializadas na feira livre de pocinhos - PB. 2012. 35 f. TCC (Gradua-ção) - Curso de Biologia, Universidade Estadual da Paraíba, Campina Grande, 2012.

CRISTINE, A. et al. Análise das Condições do Comércio de Caldo de Cana em Vias Públicas de Municípios Paulistas. Segurança Alimentar e Nutricional, v. 13, n. 2, p. 6-18, 2006.

ESTEVESA, Fabrício Andrade Martins; FIGUEIRÔA, Evellyne de Oliveira. Detecção de enteroparasitas em hortaliças comercializadas em feiras livres do município de Caruaru (PE). Revista Baiana de Saúde Publica, Salvador, v. 2, n. 33, p.184-193, abr./jun. 2009. 
LIMA, C. DE; SAMPAIO, M. G. V. Isospora Belli: Uma revisão de literatura. Centro Universitário Católica de Quixadá, v. 1, n. 88, 2016.

LOPES, G.; CRESTO, R.; CARRARO, C. N. M. Análise microbiológica de caldos de cana comercializados nas ruas de Curitiba, PR. Higiene Alimentar, n.147, v.20, p.40-44, 2007.

LUZ, João Gabriel Guimarães et al. Contamination by intestinal parasites in vegetables marketed in an area of Jequitinhonha Valley, Minas Gerais, Brazil. Revista de Nutrição, [s.l.], v. 30, n. 1, p.127-136, fev. 2017. FapUNIFESP (SciELO).

NEVES, D. P.; MELO, A. L.; LINARDI, P. M. Parasitologia Humana. 11. ed. Rio de Janeiro: Atheneu, 2005.

OLIVEIRA, U. D. de; JUREMA, S.; CHIUCHETTA, R. Ocorrência de enteroparasitose na população do município de Goierê, PR. PR, 31, 81-85, 2009.

PEREIRA, A. C. M. (2016). Detecção dos protozoários Giardia lamblia e Cryptosporidium sp. em saladas pré-embaladas, 95. Acesso em: 14 de maio de 2018. Disponível em: https://estudogeral.sib.uc.pt/bitstream/10316/40731/1/AdelaideCarina.pdf.

PRADO, S. DE P. T. et al. Avaliação do perfil microbiológico e microscópico do caldo de cana in natura comercializado por ambulantes. Revista Instituto Adolfo Lutz, São Paulo - SP, v. 69, n. 1, p. 55-61, 2010.

REY, L. Parasitologia. 4. ed. Rio de Janeiro: Guanabara Koogan, 2008.

ROSA, G.; JULIÃO, V. A. P.; MESSA, V.; MAGALHÃES, R.; MERLINI, L. S. Pesquisa de entamoeba hystolytica e ancylostoma duodenale em manipuladores de alimentos do município de Umuarama, Paraná. v. 43, n. 4, p. 1-32, 2010.

SILVA, L. B. . Uma abordagem sobre as principais parasitoses intestinais mais prevalentes na infância e suas causas. Centro Universitário São Lucas - Porto Velho, p. 1-63, 2017.

\section{Como citar este artigo (Formato ABNT):}

PINA, Frederico A. N.; ESPINHEIRA, Marcelo J. C.L.; SOUZA, Flávio M. de. Análise Parasitológica de Caldos de Cana Comercializados em Feiras Livres em uma Cidade no Interior da Bahia. Id on Line Rev.Mult. Psic., 2018, vol.12, n.40, p.859-869. ISSN: 1981-1179.

Recebido: 23/05/2018

Aceito: $24 / 05 / 2018$ 